between them. Caesar et al. take the top-down approach: their inferences of changes in the AMOC strength are made from reconstructions of regional and global SSTs that are derived from direct measurements of temperature. It is possible that regions other than the North Atlantic in which there has been decadal-scale variability in SSTs could influence the mean global SST from which the AMOC strength is calculated - although the authors do attempt to quell such doubts by showing that the subpolar-gyre SST anomaly is robust relative to the global mean SST for a subset of time periods (see Extended Data Fig. 2 in ref. 4).

Thornalley and colleagues' strategy is more of a bottom-up approach: they use a proxy for deep-water current strength to measure AMOC strength more directly than do Caesar and co-workers. The weaknesses of this approach are that it accounts for only the local bottom currents at the sites from which the cores are taken, which might not capture the entire AMOC system, and that it could be susceptible to local nonlinear effects such as abrupt shifts in the position of the current. However, Thornalley et al. show that there is a striking correlation between their

grain-size proxy and the measured density of the Labrador Sea Water (a major component of the North Atlantic Deep Water), as well as with the heat content of the subpolar gyre; these correlations shore up the bridge that links their localized proxy measurements to broader-scale changes in the AMOC.

For now, the timing of the AMOC decline remains a source of intrigue. Future studies

"The two estimates of the decline are remarkably similar, despite the different time periods on which they are based." that provide a moredetailed spatial network of multiple proxy reconstructions will help to clarify some of the remaining ambiguity about which elements of the AMOC were changing and when. It is - at least scientifically - reassuring to see that the present two studies converge on the conclusion that the modern AMOC is in a relatively weak state. However, in the context of future climate-change scenarios and a possible collapse in the $\mathrm{AMOC}^{11}$ in response to the continued melting of the Greenland Ice Sheet ${ }^{12}$, it is perhaps less reassuring, because a weakened
AMOC might lead to considerable changes in climate and precipitation patterns throughout the Northern Hemisphere ${ }^{13}$.

Summer K. Praetorius is in the Geology, Minerals, Energy, and Geophysics Science Center, US Geological Survey, Menlo Park, California 94025, USA.

e-mail:spraetorius@usgs.gov

1. Broecker, W. S. Oceanography 4, 79-89 (1991).

2. Henry, L. G. et al. Science 353, 470-474 (2016).

3. Srokosz, M. A. \& Bryden, H. L. Science 348, 1255575 (2015).

4. Caesar, L., Rahmstorf, S., Robinson, A., Feulner, G. \& Saba, V. Nature 556, 191-196 (2018).

5. Thornalley, D. J. R. et al. Nature 556, 227-230 (2018).

6. Drijfhout, S., van Oldenborgh, G. J. \& Cimatoribus, A J. Clim. 25, 8373-8379 (2012).

7. Rahmstorf, S. et al. Nature Clim. Change 5, 475-480 (2015).

8. Menary, M. B. \& Wood, R. A. Clim. Dyn. 50, 3063-3080 (2017).

9. McCave, I. N., Manighetti, B. \& Robinson, S. G. Paleoceanography 10, 593-610 (1995).

10.PAGES 2k Consortium. Nature Geosci. 6, 339-346 (2013).

11.Liu, W., Xie, S.-P., Liu, Z. \& Zhu, J. Sci. Adv. 3, e1601666 (2017).

12.Bakker, P. et al. Geophys. Res. Lett. 43, 12252-12260 (2016).

13.Jackson, L. C. et al. Clim. Dyn. 45, 3299-3316 (2015).

\title{
The telomerase enzyme and liver renewal
}

Cell-tracing analysis reveals that a disperse group of cells in the mouse liver express the enzyme telomerase, which preserves chromosome ends. These cells contribute to liver maintenance and regeneration. SEE LETTER P.244

\section{KENNETH S. ZARET}

$\mathrm{T}$ The enzyme telomerase maintains the length of specialized repetitive structures called telomeres, which are found at the ends of chromosomes. When they become damaged or shortened, telomeres can stop cells from dividing ${ }^{1}$. Most cells in adult humans have very low or undetectable levels of telomerase and relatively short telomeres, and therefore have a limited ability to replicate $^{2}$. However, elevated telomerase levels are seen in various animal and human stem cells that must retain their replicative capacity for self-renewal ${ }^{3}$. Telomerase defects are associated with tissue scarring (fibrosis) in the livers of both mice and humans ${ }^{4,5}$, but which cells in the liver express telomerase, and whether they act as stem cells, has been unclear. On page 244 , Lin et al. ${ }^{6}$ characterize this cell population in mice.

First, the authors identified telomeraseexpressing cells in the mouse liver and tracked descendent cells. The group genetically engineered mice to contain a modified version of the gene Tert, which encodes a subunit of telomerase. When the mice are treated with a drug, this alteration causes cells expressing Tert to be indelibly labelled by a fluorescent protein. Once the genetically modified cells are triggered in this way, they and all their descendants produce the fluorescent protein, even if the cells no longer express Tert itself.

Lin et al. found that 3-5\% of hepatocytes, the most prevalent type of cell in the liver, fluoresce in response to drug treatment. The authors confirmed, by quantitation of messenger RNA levels, that these cells express Tert. Next, they examined the livers of adult mice one year after drug treatment. The initially labelled cells (dubbed Tert ${ }^{\text {High }}$ ) had given rise to clusters of descendants dispersed throughout the liver's lobes, making up about $30 \%$ of the liver's total mass (Fig. 1). Adult hepatocytes die and are replaced infrequently, so the increase in labelled cells over long periods indicates that the Tert ${ }^{\text {High }}$ hepatocytes contribute to the gradual renewal of the liver under normal conditions.

A key question is whether the Tert ${ }^{\text {High }}$ hepatocytes are a stable, self-renewing population. Alternatively, Tert could be expressed in certain cells for a period of time, then shut off in those hepatocytes and expressed in others. In support of the former case, when Lin et al. triggered fluorescent-protein labelling three times over a ten-week period, they found that the numbers of labelled hepatocytes were comparable to those for a single trigger. Next, they showed that $75 \%$ of labelled hepatocytes expressed high levels of Tert mRNA when they were examined a month after a single drug treatment, whereas only $18 \%$ did so after a year, indicating that, as the population gradually expands, Tert ${ }^{\text {High }}$ cells not only self-renew but also give rise to progeny that do not express Tert $\left(\right.$ Tert $\left.{ }^{\mathrm{Low}}\right)$. Finally, the researchers demonstrated that Tert ${ }^{\text {High }}$ hepatocytes proliferate more than Tert ${ }^{\text {Low }}$ cells, whereas Tert ${ }^{\text {Low }}$ cells exhibit higher expression of genes relating to metabolism and biosynthesis than do Tert ${ }^{\text {High }}$ cells.

Taking these data together, the authors suggest that Tert ${ }^{\text {High }}$ hepatocytes behave like stem cells. But before concluding that the Tert ${ }^{\text {High }}$ cells are bona fide stem cells for the liver, it will be necessary to determine whether the Tert ${ }^{\text {High }}$ population becomes exhausted or remains at similar levels in older mice (because hepatocytes are still renewed in ageing mice), and whether Tert ${ }^{\text {Low }}$ cells convert to Tert ${ }^{\text {High }}$ over longer periods than those used here (which would indicate that this population is not acting as stem cells). It will also be interesting 

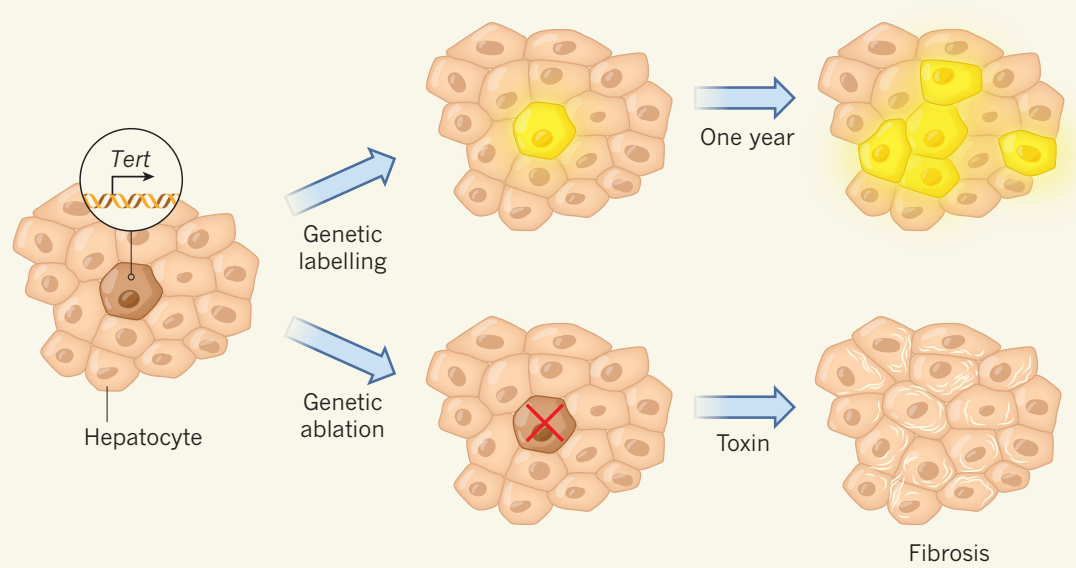

Figure 1 | Lineage tracing in the liver. Lin et al. ${ }^{6}$ characterize the hepatocyte cells in the mouse liver that express high levels of the gene Tert, which encodes a subunit of the enzyme telomerase. The authors generated mice that carry a genetically engineered version of Tert: when the mice are treated with a drug, any cells expressing Tert are indelibly labelled with a fluorescent protein. Those cells and all their descendants fluoresce, and so can be tracked. Only 3-5\% of cells fluoresced immediately after drug treatment. One year later, about $30 \%$ of cells fluoresced, but most of these did not express Tert, indicating that the rare Tert-expressing cells give rise to new hepatocytes to help regenerate the liver. If the Tertexpressing cells are genetically ablated, the liver is susceptible to scarring (fibrosis) after toxin damage.

to determine the processes by which cells transition from Tert ${ }^{\text {High }}$ to Tert ${ }^{\text {Low }}$, and how this change relates to homeostatic control of liver mass.

Importantly, stem cells typically reside in a special tissue compartment, or niche, that supports their regenerative capacity. Yet the Tert $^{\text {High }}$ cells are dispersed throughout the liver. This dispersal of Tert ${ }^{\text {High }}$ cells is interesting because hepatocytes reside in different zones in each lobe of the liver, and earlier studies ${ }^{7}$ implicated one zone or another as being more relevant to liver regeneration. By contrast, Lin et al. provide evidence for a 'distributed model' for hepatocyte renewal. The research indicates that, although the Tert ${ }^{\text {High }}$ hepatocytes possess features of stem cells, those features are not of a conventional type.

In the past three years, one regenerative hepatocyte population near the central vein has attracted particular attention. The population responds to venous signals to self-renew during homeostasis, producing progeny that migrate outwards from the central zone ${ }^{8}$. Lin et al. found a few Tert ${ }^{\text {High }}$ hepatocytes in the central zone in healthy livers, but these cells did not reside close enough to the central vein to respond to its signals. However, when the authors damaged the central-vein zone, Tert ${ }^{\text {High }}$ descendants appeared there and responded to venous signals. Moreover, after damage to the liver tissue in another region, around the portal vein, hepatocytes descended from Tert ${ }^{\text {High }}$ cells appeared abundantly in the periportal and mid-lobular zones, and the researchers found that ablation of Tert ${ }^{\text {High }}$ hepatocytes impaired this regenerative response, leading to liver fibrosis. Taking the above findings together with those of other studies of liver injury, it seems that various types of hepatocyte (as well as cells from the bile duct $)^{9-12}$ can regenerate the mouse liver under a range of damage conditions.

In the future, it will be crucial to assess how relevant these findings in mice are to human liver regeneration. The fact that ablation of Tert ${ }^{\text {High }}$ hepatocytes results in fibrosis in the injured mouse liver seems to support relevance for humans, because people who harbour mutations in TERT and genes that encode other telomere-related factors can also exhibit fibrosis and cirrhosis (the latter being a predictor of liver cancer) ${ }^{5}$. However, Tert ${ }^{\text {High }}$ hepatocytes have not been seen in human livers - although the possibility has not yet been assessed with the sensitivity of the genetic-labelling approach used in mice by Lin and colleagues. An alternative explanation for diseases in humans who have telomerase-related mutations is that excessive telomere shortening in early development might affect many organ progenitors in a nonspecific way.

More-detailed studies in humans will be needed to confirm how telomerase-based regeneration forestalls liver disease, and possibly liver cancer. Nevertheless, Lin and colleagues' study provides insight into a previously unidentified, dispersed-cell mode of liver regeneration.

Kenneth S. Zaret is in the Department of Cell and Developmental Biology, Institute for Regenerative Medicine, Perelman School of Medicine, University of Pennsylvania, Philadelphia, Pennsylvania 19104, USA. e-mail:zaret@pennmedicine.upenn.edu

1. Palm, W. \& de Lange, T. Annu. Rev. Genet. 41, 301-334 (2008).

2. Blasco, M. A. Nature Chem. Biol. 3, 640-649 (2007).

3. Günes, C. \& Rudolph, K. L. Cell 152, 390-393 (2013).

4. Rudolph, K. L., Chang, S., Millard, M., Schreiber-Agus, N. \& DePinho, R. A. Science 287, 1253-1258 (2000).

5. Calado, R. T. et al. PLoS ONE 4, e7926 (2009).

6. Lin, S. et al. Nature 556, 244-248 (2018).

7. Stanger, B. Z. Annu. Rev. Physiol. 77, 179-200 (2015).

8. Wang, B., Zhao, L., Fish, M., Logan, C. Y. \& Nusse, R. Nature 524, 180-185 (2015).

9. Yanger, K. et al. Cell Stem Cell 15, 340-349 (2014).

10.Tarlow, B. D. et al. Cell Stem Cell 15, 605-618 (2014).

11.Font-Burgada, J. et al. Cell 162, 766-779 (2015).

12. Raven, A. et al. Nature 547, 350-354 (2017).

This article was published online on 4 April 2018.

\section{EVOLUTION}

\section{Backbone of RNA viruses uncovered}

The evolutionary history of viruses is largely unknown. Large-scale discovery of vertebrate RNA viruses shows that, although viruses often jump between hosts, most have co-evolved with their hosts over millions of years. SEE ARTICLE P.197

\section{MARK ZELLER \& KRISTIAN G. ANDERSEN}

$\mathrm{M}$ any human diseases, from the common cold to deadly haemorrhagic fevers, are caused by RNA viruses. Most of these viruses are thought to have originated from close relatives that infected mammals ${ }^{1,2}$, and so the majority of virus-discovery studies have focused on mammals and birds ${ }^{3}$. RNA viruses, however, are probably older than the last common ancestor of life on Earth ${ }^{4,5}$. Detailed genetic information for RNA viruses from other classes of vertebrate is sorely needed if we are to fully understand long-term virus evolution. On page 197, Shi et al. ${ }^{6}$ report the discovery of previously unidentified vertebrate RNA viruses from across evolutionary timescales.

The authors analysed the viruses in 186 vertebrate species using an approach called metatranscriptomic sequencing, in which all of the RNA present in a sample is sequenced. The samples were taken from 